\title{
Successful Treatment of a Paroxysmal Kinesigenic Dyskinesia Patient with Carbamazepine-Induced Stevens-Johnson Syndrome Using Oxcarbazepine Monotherapy: A Case Report
}

\author{
Hung T. Tran ${ }^{a, b} \quad$ Khang V. Nguyen ${ }^{a}$ Laurent Vercueil ${ }^{c, d}$ \\ aDepartment of Neurology, University of Medicine and Pharmacy at Ho Chi Minh City, \\ Ho Chi Minh City, Vietnam; bParkinson's disease and Movement disorders clinic, Nguyen \\ Tri Phuong Hospital, Ho Chi Minh City, Vietnam; 'Exploration Fonctionnelle du Système \\ Nerveux, Pôle de Psychiatrie, Neurologie et Rééducation Neurologique, Centre Hospitalier \\ Universitaire (CHU) Grenoble, Grenoble, France, ${ }^{d}$ INSERM U836, Grenoble Institut des \\ Neurosciences, La Tronche, France
}

Keywords

Antiepileptic drugs · Case report · Paroxysmal kinesigenic dyskinesia · Stevens-Johnson syndrome

\begin{abstract}
Paroxysmal kinesigenic dyskinesia (PKD) is a rare condition characterized by abnormal involuntary movements that are precipitated by a sudden movement. PKD is often misdiagnosed with psychogenic movement disorders. Carbamazepine is usually the first choice of medication due to its well-established evidence but could induce Stevens-Johnson syndrome. We report a 21-year-old male patient with PKD referred to our movement disorders clinic after being misdiagnosed with conversion syndrome. PRRT2 gene testing using next-generation sequencing revealed a mutation in c.649dupC p. (Arg217fs). The patient responded well to carbamazepine but had to withdraw the treatment due to carbamazepine-induced StevensJohnson syndrome after 3 weeks of medication. Our patient did not respond to trials of levetiracetam and phenytoin but finally responded well to oxcarbazepine. The patient was followed up for 4 years, during which he had no attacks and no side effects. Here, we present a PKD case with carbamazepine-induced Stevens-Johnson syndrome successfully treated with
\end{abstract}


Tran et al.: Treatment of PKD with Carbamazepine-Induced Stevens-Johnson Syndrome

oxcarbazepine despite the risk of cross-reactive skin eruption between these antiepileptics. Careful history taking and examining patient's attacks are crucial to accurate diagnosis and treatment in PKD patients.

(C) 2021 The Author(s).

Published by S. Karger AG, Basel

\section{Introduction}

Paroxysmal dyskinesia is a group of paroxysmal movement disorders consisting of paroxysmal kinesigenic, nonkinesigenic, and exercise-induced dyskinesia. Paroxysmal kinesigenic dyskinesia (PKD) is characterized by hyperkinetic movements that occur after sudden movement, acceleration, or intention to move. PKD manifestation consists of dystonia, choreoathetosis, ballismus, or a combination of those hyperkinetic movements [1]. Genetic testing often reveals mutations in proline-rich transmembrane protein (PRRT2), modulating the $\mathrm{Na}^{+}$ channel in neurons. Brain MRI and EEG often show no abnormalities in patients with PKD. Due to its uncommon symptoms and unrevealing routine diagnostic tests, PKD is often mistaken for psychiatric disorders [2]. Carbamazepine is the first line of treatment for PKD. Despite its efficacy, carbamazepine can cause Stevens-Johnson syndrome (SJS), which is a hypersensitivity reaction causing severe dermatitis and could be life threatening [3].

Our patient was diagnosed with PKD and had an excellent response to carbamazepine. However, carbamazepine had to be withdrawn due to SJS 3 weeks later. His symptoms were not controlled by other medications except oxcarbazepine. Therefore, we present a case with a novel treatment of PKD with SJS due to carbamazepine.

\section{Case Report/Case Presentation}

A 21-year-old man presented to our movement disorders clinic with a 12-year history of attacks of involuntary movements of both upper limbs and upper trunk. These attacks are often triggered by sudden movements such as standing up quickly or running on the spot. The patient was conscious of experiencing a certain "feeling" before the onset of an attack. The patient was able to prevent these attacks by standing up slowly. He once had a febrile seizure when he was 2 months old and was treated in Children Hospital No. 1 in Ho Chi Minh City with an unclear diagnosis and treatment. He had no family history of similar conditions, epilepsy, seizure, or other movement disorders. These attacks were increased in frequency to $>20$ times a day, rendering him from continuing his education 3 years after symptom onset. His parents thought the symptoms were related to psychological disorders, so the patient did not receive proper medical care. When he was 21 years old, he was referred to a local psychiatric hospital in Ho Chi Minh City. A brain MRI and electroencephalogram were done, but no abnormal sign was found. He was diagnosed with "conversion disorder." He was prescribed amisulpride $400 \mathrm{mg} 1 \frac{1}{2}$ tablet, BID; trihexyphenidyl 2 mg 1 tablet, BID; and valproate magnesium $200 \mathrm{mg} 1$ tablet, BID, for 2 months, but there was not any amelioration. He then withdrew medications for 6 months, and the symptoms unchanged.

Finally, he came to our Parkinson's disease and Movement disorders clinic at Nguyen Tri Phuong Hospital. Careful history taking and clinical examination showed no signs of Parkinsonism, ataxia, and no focal neurologic deficits or psychiatric symptoms including delusions, hallucinations, disorientation, and emotional volatility. Therefore, PKD was suspected. However, we could not observe the attack at our clinic due to the transient and unpredictable nature of the attacks. We then instructed the patient to self-record the attack constantly athome. After observing

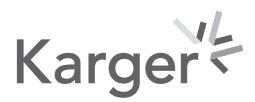


Tran et al.: Treatment of PKD with Carbamazepine-Induced Stevens-Johnson Syndrome

Fig. 1. Front view of the patient's body.

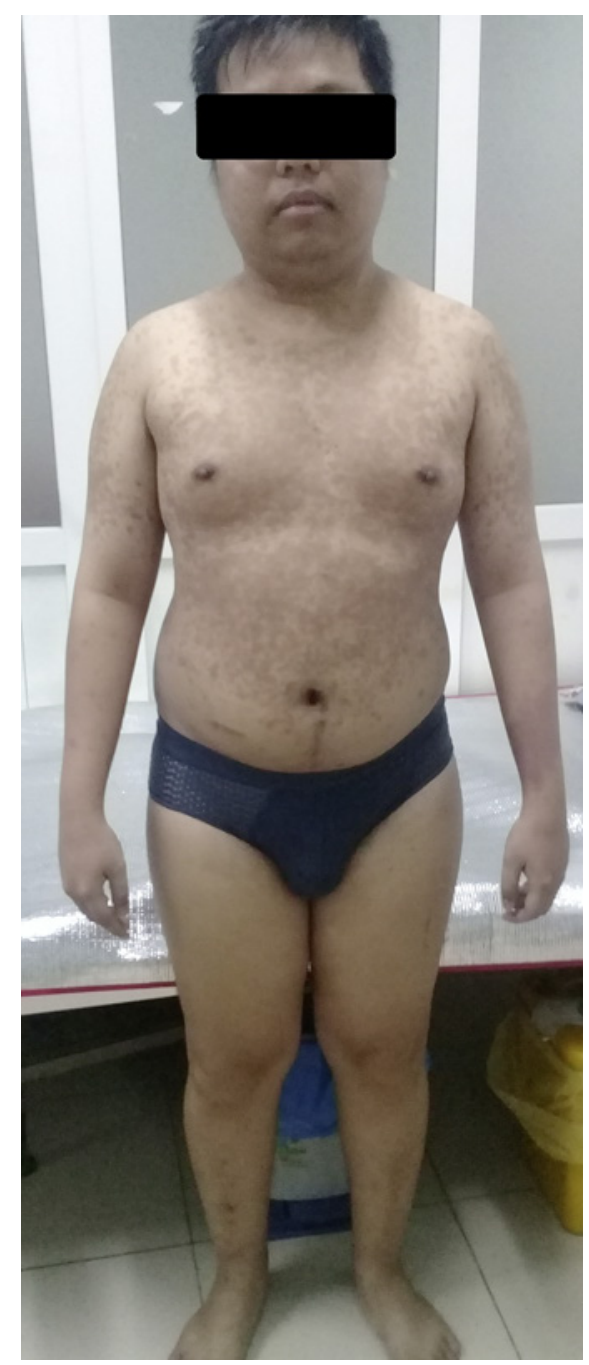

and analyzing the patient's video, we witnessed the manifestations of the attack, including choreic movements and dystonic posture of the upper limbs, head, neck, and upper part of the trunk. The patient usually had to hold on to the nearest table to decrease the magnitude of movements. The duration of each attack was about 10-30 s. The patient's consciousness was preserved before, during, and after the attacks. There were no neurologic deficits after the attacks (see online suppl. Video 1; for all online suppl. material, see www.karger.com/doi/10.1159/000518891).

Nevertheless, PRRT2 gene testing was a relatively new test in Vietnam, so it took us a few months to get the test result. The next-generation sequencing test was done by using NextSeq 550 System Guide - Illumina Inc. at Medical Genetics Institute in Ho Chi Minh City, which later revealed a mutation in c.649dupC p. (Arg217fs). Therefore, PKD with PRRT2 mutation was our final diagnosis. After a few days of carbamazepine $200 \mathrm{mg} 1 / 2$ tablet, BID prescription, the patient no longer suffered from any attack. Twenty days later, he developed skin and mucous membrane eruptions. Following a dermatologist consultation, he was diagnosed with carbamazepineinduced SJS (shown in Fig. 1-4). Therefore, carbamazepine was withdrawn, and the patient's SJS skin and mucous membrane lesions made a self-recovery without specific treatment. HLA-B gene testing was done using next-generation sequencing, but the HLA-B*1502 allele was not found. After unsuccessful trials of levetiracetam $500 \mathrm{mg}$ BID and phenytoin $100 \mathrm{mg}$ TID monotherapy, the patient finally responded well to oxcarbazepine $300 \mathrm{mg}$ tablet, BID, with no attack. 
Tran et al.: Treatment of PKD with Carbamazepine-Induced Stevens-Johnson Syndrome

Fig. 2. Back view of the patient's body.

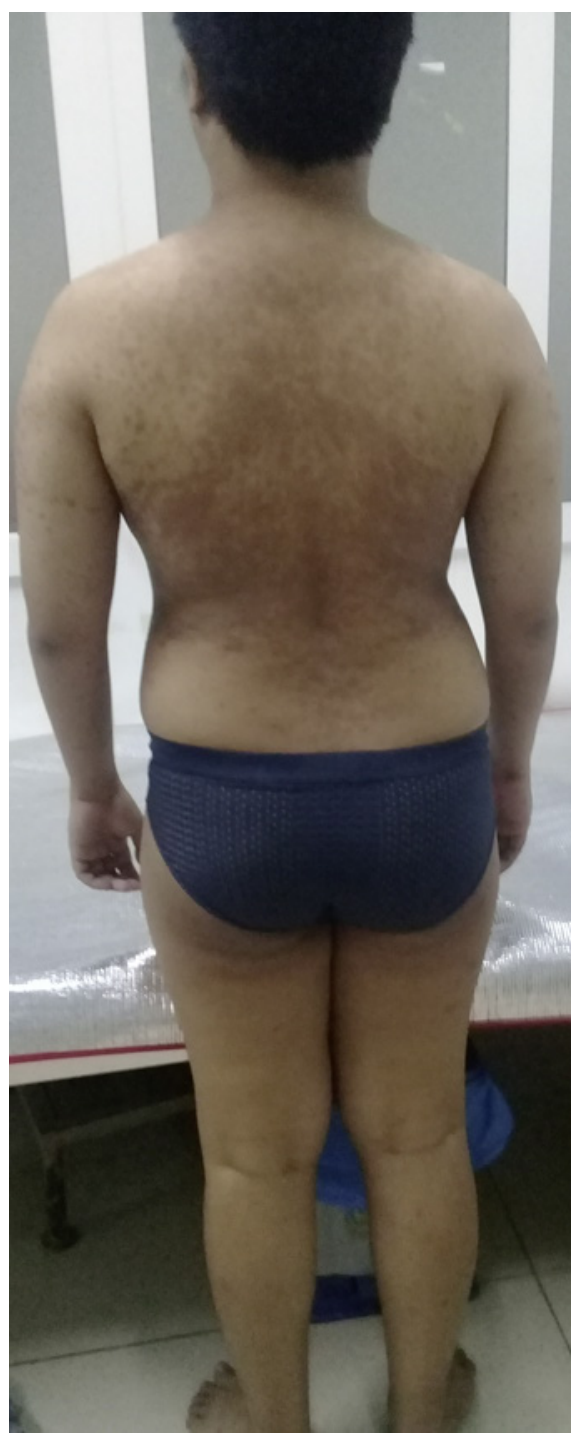

He was followed up to 4 years at our movement disorders clinic, and oxcarbazepine dose was decreased to $300 \mathrm{mg} 1$ tablet a day in the morning. During the follow-up period, the patient had no attacks, no side effects, and no psychiatric symptoms and can continue his education. He now has a normal life and is pleased with the current treatment. However, when he forgets a dose, his symptoms re-emerge.

\section{Discussion/Conclusion}

PKD is commonly misdiagnosed due to its unfamiliar clinical presentation and unrevealing signs in the routine workup, including EEG and brain MRI. Cases are also often sporadic, and no patient's relatives have similar symptoms [4]. Definite diagnosis is made by genetic testing, which often reveals mutations in PRRT2 in the long arm of chromosome 16 [5]. Our patient showed no sign of psychiatric symptoms but showed typical symptoms of PKD on history and patient's self-video-recording. Therefore, psychiatrists, general neurolo- 
Tran et al.: Treatment of PKD with Carbamazepine-Induced Stevens-Johnson Syndrome

Fig. 3. Palm view of the patient's hand.

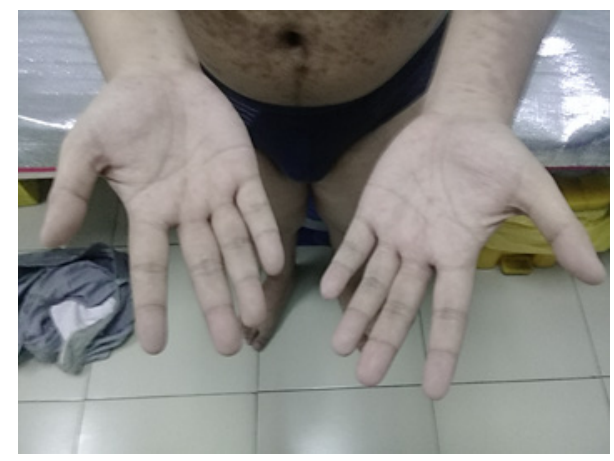

Fig. 4. A closer image at the patient's trunk.

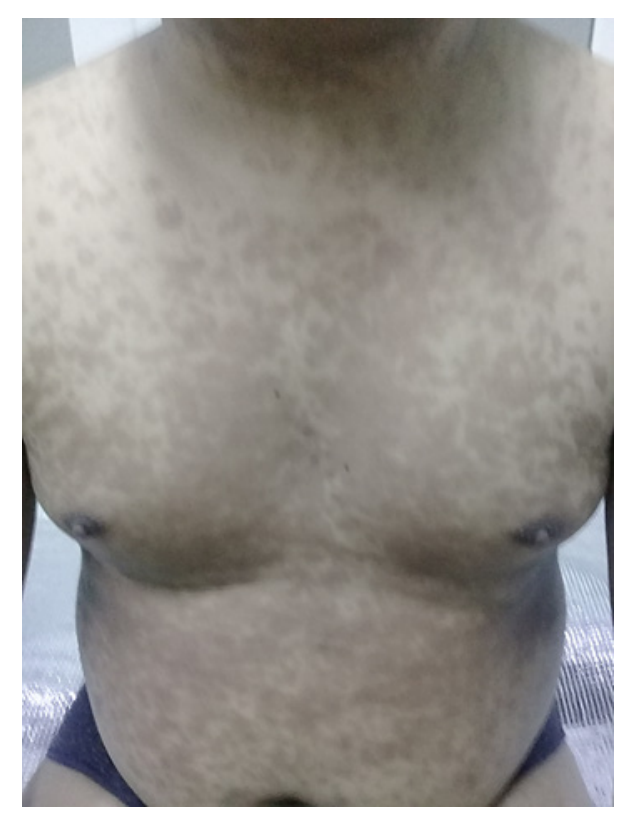

gists, and primary care clinicians need to recognize PKD's unusual symptoms to refer patients to movement disorders specialists. Our patient was on valproate magnesium $400 \mathrm{mg}$ and amisulpride $400 \mathrm{mg}$ for 2 months. Valproate could cause Parkinsonism, but this side effect would cease after drug withdrawal. Amisulpride could cause extrapyramidal symptoms, including akathisia, Parkinsonism, acute dystonia, or tardive dyskinesia [6]. Tardive dyskinesia is a hyperkinetic movement disorder with slow, persistent, involuntary, and repetitive body movements in the face, lips, tongue, trunk, and extremities that often occur in patients with prolonged use of antipsychotics [7]. However, to our knowledge, no case of amisulpride causing paroxysmal dyskinesia was reported.

Treatment of PKD is only limited to symptomatic treatment, but patients usually experience complete relief of symptoms [4]. Carbamazepine is a well-established first-line treatment but can carry the risk of SJS, which is a severe delayed-type hypersensitivity reaction [3]. Many other antiepileptic drugs could also cause SJS, including phenytoin, lamotrigine, and oxcarbazepine [8]. Asian ethnicity has a higher risk of SJS because they often carry the HLA-B*1502 allele [9]. Although our patient does not carry the HLA-B*1502 allele, he had SJS during his treatment, making him stop using carbamazepine. Therefore, a patient without the HLA-B*1502 allele is not without risk of SJS [10].

There also have been case reports on other effective anticonvulsants for PKD, including phenytoin, valproate, gabapentin, lamotrigine, levetiracetam, and phenobarbital [11]. 
However, our patient did not respond to phenytoin $100 \mathrm{mg}$ TID or levetiracetam $500 \mathrm{mg}$ BID monotherapy. He responded well to monotherapy of oxcarbazepine $300 \mathrm{mg}$ per day. Other reported cases also responded to low doses, between 75 and $300 \mathrm{mg}$ oxcarbazepine per day [12]. Despite the risk of cross-reactive skin eruption between carbamazepine and oxcarbazepine [13] and the report of many cases of oxcarbazepine-induced SJS [14], our patient did not experience any side effect during his oxcarbazepine monotherapy up until now.

A particular strength of our study is that our PKD patient, in whom diagnosis was confirmed by next-generation sequence testing, was followed up continuously from 2017 to 2021. This long follow-up period allows us to monitor the patient's medication response and any side effects. Our study also has limitations. Because this is a case report, our treatment of PKD with SJS could not be generalized without more evidence from randomized controlled trials. However, our study has provided some background for the design of future study.

In this case report, we want to emphasize the importance of clinical suspicion of PKD not to be misdiagnosed with psychiatric disorders. To the authors' knowledge, no case of PKD confirmed by genetic testing with SJS due to carbamazepine successfully treated with oxcarbazepine was reported. We hope this case report would aid physicians in their treatment of PKD patients.

\section{Statement of Ethics}

Written informed consent was obtained from the patient to publish this case report and any accompanying images and video. The patient was followed up at our movement disorders clinic. The study is exempt from ethics committee approval because the study is observational. The conduct of this study did not affect the patient treatment course.

\section{Conflict of Interest Statement}

The authors declare that there are no conflicts of interest.

\section{Funding Sources}

The authors received no financial support for the research, authorship, and publication of this article.

\section{Author Contributions}

T.T.H. is the chief investigator who examined, made the diagnosis, and treated the patient. He also wrote and edited the manuscript. N.V.K. and L.V. made substantial contributions to drafting and revising the manuscript. All authors read and approved the final manuscript.

\section{Data Availability Statement}

All data generated or analyzed during this study are included in this article and its online suppl. material files. Further enquiries can be directed to the corresponding author. 


\section{References}

1 Erro R, Sheerin UM, Bhatia KP. Paroxysmal dyskinesias revisited: a review of 500 genetically proven cases and a new classification. Mov Disord. 2014;29(9):1108-16.

2 Pan F, Li S, Li H, Xu Y, Huang M. Misdiagnosed atypical paroxysmal kinesigenic dyskinesia: a case report. Neuropsychiatr Dis Treat. 2018;14:1433-5.

3 Ferrell PB Jr, Carbamazepine MLHL. HLA-B*1502 and risk of Stevens-Johnson syndrome and toxic epidermal necrolysis: US FDA recommendations. Pharmacogenomics. 2008;9(10):1543-6.

4 Unterberger I, Trinka E. Diagnosis and treatment of paroxysmal dyskinesias revisited. Ther Adv Neurol Disord. 2008;1(2):4-11.

5 Huang XJ, Wang T, Wang JL, Liu XL, Che XQ, Li J, et al. Paroxysmal kinesigenic dyskinesia: clinical and genetic analyses of 110 patients. Neurology. 2015;85(18):1546-53.

6 Martino D, Karnik V, Osland S, Barnes TRE, Pringsheim TM. Movement disorders associated with antipsychotic medication in people with schizophrenia: an overview of cochrane reviews and meta-analysis. Can J Psychiatry. 2018;63(11). Epub ahead of print.

7 Cornett EM, Novitch M, Kaye AD, Kata V, Kaye AM. Medication-induced tardive dyskinesia: a review and update. Ochsner J. 2017;17(2):162-74.

8 Sukasem C, Katsila T, Tempark T, Patrinos GP, Chantratita W. Drug-induced Stevens-Johnson syndrome and toxic epidermal necrolysis call for optimum patient stratification and theranostics via pharmacogenomics. Annu Rev Genomics Hum Genet. 2018;19:329-53.

9 Tangamornsuksan W, Chaiyakunapruk N, Somkrua R, Lohitnavy M, Tassaneeyakul W. Relationship between the HLA-B*1502 allele and carbamazepine-induced Stevens-Johnson syndrome and toxic epidermal necrolysis: a systematic review and meta-analysis. JAMA Dermatol. 2013;149(9):1025-32.

10 Shi YW, Min FL, Qin B, Zou X, Liu XR, Gao MM, et al. Association between HLA and Stevens-Johnson syndrome induced by carbamazepine in Southern Han Chinese: genetic markers besides B*1502? Basic Clin Pharmacol Toxicol. 2012;111(1):58-64.

11 Strzelczyk A, Bürk K, Oertel WH. Treatment of paroxysmal dyskinesias. Expert Opin Pharmacother. 2011; 12(1):63-72.

12 Yang Y, Su Y, Guo Y, Ding Y, Xu S, Jiang Y, et al. Oxcarbazepine versus carbamazepine in the treatment of paroxysmal kinesigenic dyskinesia. Int J Neurosci. 2012;122(12):719-22.

13 Beran RG. Cross-reactive skin eruption with both carbamazepine and oxcarbazepine. Epilepsia. 1993;34(1): 163-5.

14 Beken B, Can C, Örencik A, Can N, Yazıcıoğlu M. Oxcarbazepine-induced Stevens-Johnson syndrome: a pediatric case report. Oxf Med Case Rep. 2017;2017(6):omx028. 